\title{
Les mécanismes de résiliences des ménages dans le contexte de la crise sanitaire de la COVID-19 au Mali
}

\author{
Ousmane Younoussa MAIGA ${ }^{1}$, Babakar SOUMARE ${ }^{2}$ \\ ${ }^{1}$ Université des Sciences Sociales et de Gestion de Bamako (USSGB), Faculté des Sciences Economiques et de Gestion \\ (FSEG) \\ ${ }^{2}$ Université des Sciences Sociales et de Gestion de Bamako (USSGB), Faculté des Sciences Economiques et de Gestion
} (FSEG)

\begin{abstract}
Résumé : Le présent article fait un focus sur les effets de la pandémie de la COVID-19 à travers les mesures barrières édictées pour stopper sa propagation. Il met en évidence les conséquences de la COVID-19 sur la récession économique, la transition des ménages dans la pauvreté et les stratégies de résilience utilisées contre la COVID-19. En effet, la contraction des industries productives a entrainé une baisse de 7,1 point le niveau de production. Depuis l'apparition du premier cas positif au Mali, la pandémie de la COVID-19 a durement affecté la population à travers ses conséquences notamment sur l'emploi et l'accès aux services de soins de santé et d'éducation. Les conséquences de la COVID-19 ont été plus marquées pour ses effets indirects que directs. Au niveau de l'emploi, la pandémie a entrainé une augmentation brutale des pertes d'emploi, en particulier dans les secteurs tertiaire et secondaire. Au plan de l'éducation la fermeture des écoles a contraint de garder plus de quatre millions d'enfants à la maison. Au niveau de la santé, la mobilisation des acteurs de la santé pour contrer la pandémie a conduit à des conséquences négatives sur des programmes essentiels comme la vaccination et les programmes de lutte contre certaines maladies. Environs 30 à $40 \%$ des ménages étaient dans les difficultés d'accès aux services de soins jusqu'en octobre 2020. A la même période $56 \%$ des ménages ruraux ont connues une réduction de leur revenu. Les principales stratégies d'adaptation utilisées par les ménages pour faire face aux effets de la COVID-19 outre les mesures gouvernementales ont été la vente d'actifs, l'engagement dans des activités génératrices de revenus supplémentaires, le recours à l'épargne, l'aide des amis et de la famille, la réduction de la consommation alimentaire et l'emprunt auprès des amis et de la famille. L'approche méthodologique a reposé sur une analyse descriptive des données en coupe transversale. Les données utilisées sont issues d'enquêtes réalisées par les structures relevant du système statistique nationale du Mali et des rapports d'études sur la COVID-19 au Mali.
\end{abstract}

Mots-clés : COVID-19, effets, résilience, ménages.

\section{Introduction}

Depuis l'apparition des premiers cas positifs confirmés de la COVID-19 au Mali en mars 2020, la population malienne a subi lourdement les effets sur le plan économique que social de la maladie à 
coronavirus à travers l'adoption des mesures barrières érigées par les autorités gouvernementales pour stopper sa propagation. Ces mesures étaient entre autre limitation des mouvements des personnes à travers l'instauration d'un couvre-feu nocturne entre vingt une heure et cinq heures du matin. Auquel s'ajoutaient l'interdiction des regroupements de plus de cinquante personnes avec le respect de la distanciation sociale d'un mètre au minimum, la fermeture des établissements scolaires et la fermeture des frontières terrestre et aérienne. Ces mesures ont enregistré d'énormes conséquences sur la vie quotidienne et les conditions d'existence des individus. En effet, les activités économiques, qu'elles soient du secteur formel ou informel, ont été sérieusement affectées. Pour les activités féminines, particulièrement celles inscrites dans le cadre de l'autonomisation de la femme, une étude menée par ONU Femmes sur les effets de la COVI-19 sur les femmes entrepreneures au Mali, conduit au constat que les activités menées par les femmes entrepreneures ont subi un coup suite à l'application des mesures barrières. La mise en œuvre de ces mesures a conduit beaucoup d'entre elles aux problèmes d'accès aux intrants et aux marchés, ainsi qu'à l'incapacité d'entreprendre en groupe les activités de transformation. A cet effet, environ $96 \%$ des femmes se sont vues perdre leurs emplois entre mars et avril 2020, contre seulement $4 \%$ des femmes qui ont pu maintenir ou augmenter leur production avec la pandémie à travers les activités comme la production du savon. Par ailleurs, les pertes totales estimées pour le secteur informel et les très petites entreprises appartenant aux femmes ont été estimées à environ 19 milliards de dollar. Selon les statistiques de l'Institut d'Economie Rurale (2017), le secteur agricole au Mali emploie $49 \%$ de la population agricole, entrainant la fragilisation du secteur de la transformation agro-alimentaire dominé par l'interaction, le rapprochement social et le contact physique de ceux qui y œuvrent là-dedans. La pandémie de la COVID-19 a considérablement affecté le secteur de l'emploi. En effet, la contraction de la demande mondiale, la perturbation consécutive des approvisionnements en matières premières intervenant dans la production des produits finis et intermédiaires et le couvre-feu imposé ont entrainé un ralentissement et même souvent un arrêt au pire des cas, contraignant les entreprises dans les meilleurs cas à un chômage partiel, voir le chômage massif. Ainsi, elle a induit des chocs tant du côté de l'offre et celui de la demande dont les effets majeurs se sont manifestés à travers la réduction de la disponibilité de la main-d'œuvre, les perturbations des chaînes de valeur réduisant l'offre ; en parallèle, la baisse de la consommation des ménages et le ralentissement de l'activité économique mondiale freinent la demande globale. Les secteurs ayant fortement subi les effets négatifs demeurent principalement les opérations commerciales, les ventes et l'emploi tels que révélés par l'enquête Business Pulse Survey (juin 2020). Elle fait état de $12 \%$ des entreprises contraintes à la fermeture. Les effets perturbateurs sur les opérations commerciales étaient similaires à l'ensemble des entreprises quel que soit leur emplacement géographique. Ils étaient plus marqués pour les entreprises de petites tailles et de tailles moyennes comparativement aux grandes entreprises. En effet les entreprises de petites tailles et de tailles moyennes étaient celles qui étaient susceptibles d'être enfermées dans une situation de forte réduction des recettes et de rigidité des coûts opérationnels tels que les loyers et les salaires des employés sous contrat.

L'objectif général de cette étude est d'évaluer les effets de la COVID-19 sur la vie socioéconomique des populations. De façon spécifique, il s'agit d'analyser les effets de la pandémie sur le revenu, la consommation des individus ainsi que leurs accès aux services sociaux de base tels que l'éducation, la santé.

\section{Méthodologie}

Cette analyse des mécanismes de résiliences des ménages dans le contexte de de la COVID-19 au Mali repose sur une analyse descriptive des données en coupe transversale pour évaluer dans quelle mesure 
la COVID-19 a affecté les indicateurs du développement humain. Elle fait recours aux données secondaires des enquêtes à haute fréquence (HFS) de la Banque mondiale, les bases de données de la CEDEAO sur la COVID-19 et les données du système statistique national. Ces analyses sont complétées par les données des rapports et études sur la COVID-19.

\section{Analyse de la situation de la COVID-19 et ses effets sur les indicateurs socio- économiques}

\subsection{Evolution de la pandémie dans l'Afrique de l'Ouest et au Mali}

Depuis l'apparition des premiers cas de la COVID-19 en décembre 2019 en Chine, la propagation de l'épidémie dans le monde n'a pas connu le même rythme. Contrairement aux pronostics de l'OMS, le continent africain a été celui le moins affecté par cette épidémie transformée rapidement en pandémie. Le Mali fait partie des pays ayant enregistré moins de contamination et de décès liés à la COVID-19. Les statistiques récentes indiquent 15183 cas positifs confirmés, 14285 cas guéris et 548 cas de décès de mars 2020 à octobre 2021 (ECOWAS COVID-19 Dashboard). A l'échelle nationale, le District de Bamako s'est présenté comme l'épicentre de la maladie. Cela s'explique par la concentration de la population à l'intérieur de la capitale malienne estimée à 2559044 habitants en 2020 selon les données de la Direction Nationale de la Population. En plus de Bamako les régions de Koulikoro et Tombouctou sont les plus affectées avec respectivement 2126 et 589 cas positifs. La situation épidémiologique de ces deux régions s'explique par la proximité de Koulikoro situé à une soixantaine de kilomètre du District de Bamako, et les affluences de voyages des agents de la MINUSMA ayant présenté les premiers cas positifs à Tombouctou. Les régions de Ménaka et de Taoudéni n'ont présenté aucun cas positif à nos jours.

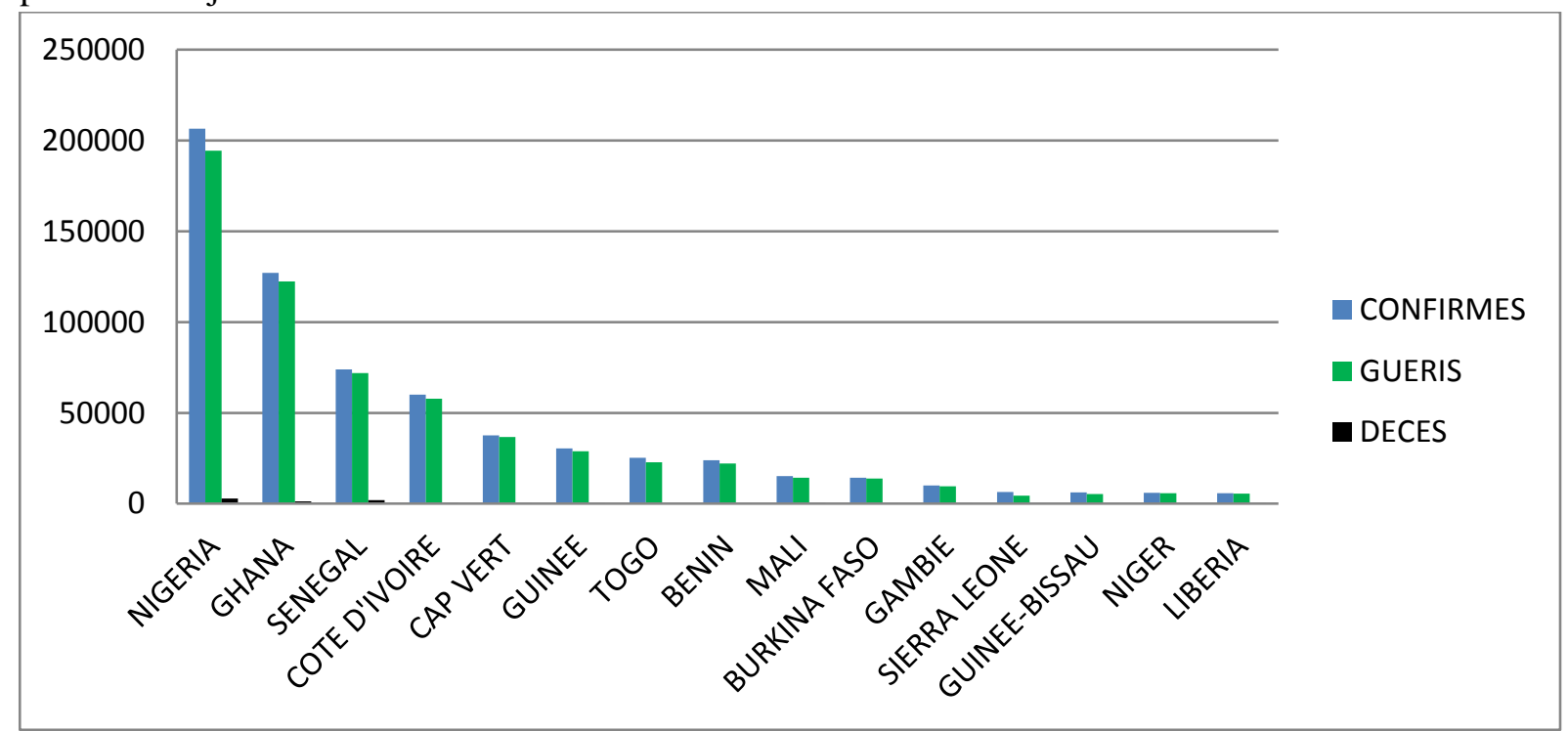

Source: ECOWAS COVID-19 Dashboard https://data.wahooas.org/outbreaks/\#/

Graphique n` 1 : Evolution de la COVID-19 en Afrique de l’Ouest 


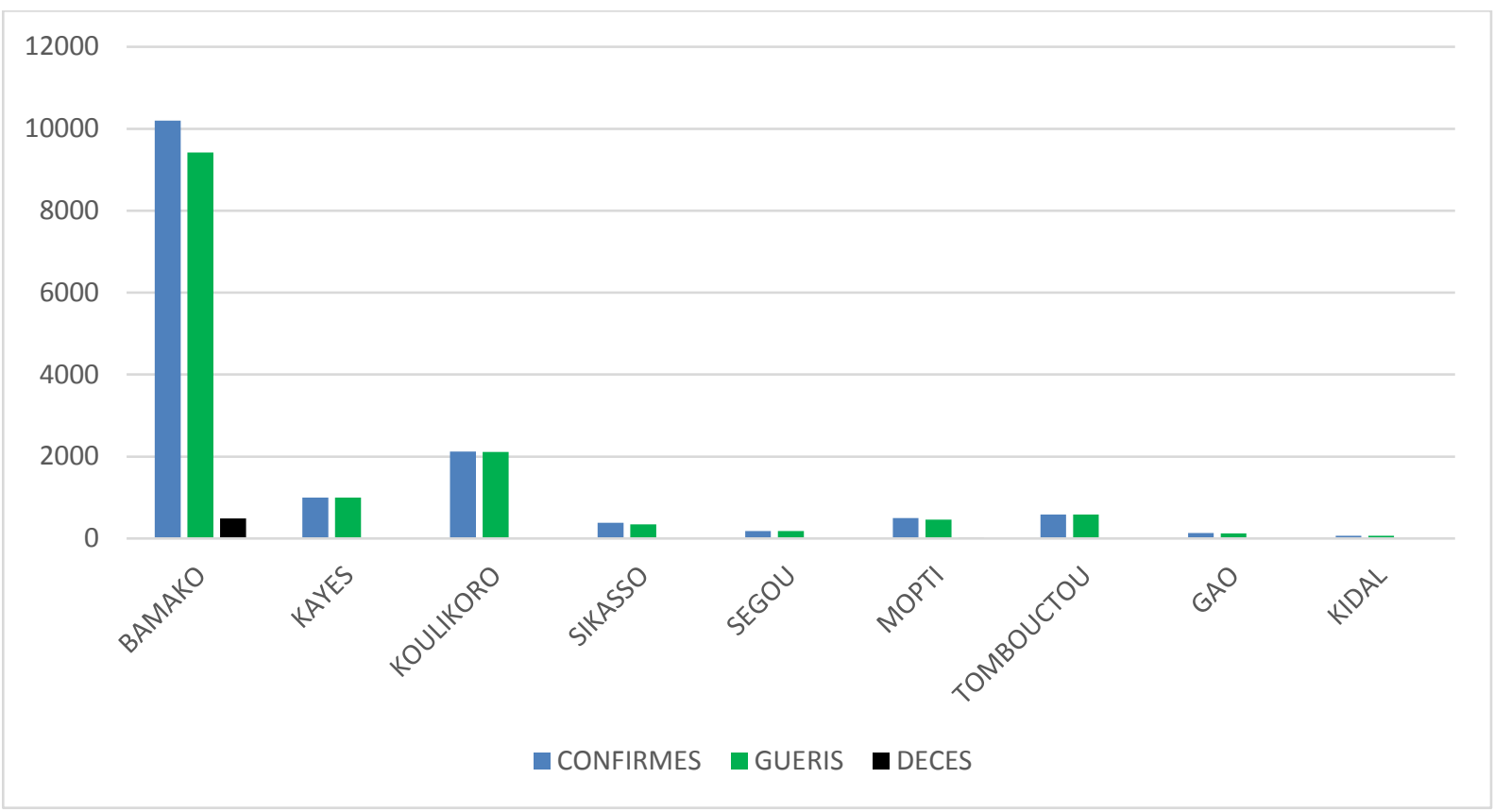

Source : ECOWAS COVID-19 Dashboard https://data.wahooas.org/outbreaks/\#/

\section{Graphique n ${ }^{\circ}$ : Evolution de la COVID-19 au Mali}

\subsection{Les effets de la COVID-19 sur les opérations commerciales}

Suivant les études de DATA Pop Alliance (2021) sur les effets socio-économiques de la COVID-19 et rôle de la gouvernance des risques de catastrophe dans le Sahel occidental, les chocs ont été inégalement répartie au sein du secteur des services. Le taux de suspension des opérations commerciales des entreprises variait entre 12 et $15 \%$. En plus des services de commerces, l'éducation, le tourisme, les restaurants et les hôtels, ont été profondément affecté par la COVID-19 en raison des mesures de confinement, y compris la fermeture des écoles, des bars et des restaurants, les couvre-feux nocturnes. En revanche, les services de santé ont connu une hausse de leurs activités tout comme les industries manufacturières où les effets n'auraient pu être visibles que dans le moyen et le long terme. La fermeture des frontières a fait payer un lourd tribut aux entreprises du secteur de l'industrie agro-alimentaire et les services de transports avec la baisse des activités commerciales et la réduction des mobilités.

Au plan mondial, les statistiques de la Banque mondiale sur les perspectives économiques mondiales (2021) indiquent une réduction de la croissance de 4,3\% en 2020 contre 2,3\% en 2019. Les principaux destinataires des exportations maliennes ont également souffert de la crise et de la contraction de l'activité qu'elle a engendrée.

En effet, la pandémie de la COVID-19 a entrainé une perturbation dans l'importation et l'écoulement de certains produits de base notamment les riz. En plus il convient de rappeler que les sanctions imposées par la CEDEAO après le coup d'État en 2020 ont contribué à amplifier les effets de la COVOD-19 sur l'économie. Suivant les constats, l'adoption des 
mesures restrictives ont contribué à une baisse de 7,1 point imputable à la contraction des industries productives et la réduction de la demande intérieure (Banque Mondiale, 2021). Sur la période couvrant mai et juillet 2020, les emplois perdus ont été plus importants dans les secteurs du commerce, de l'agriculture, des services personnels et de l'éducation. Les administrations publiques et les services de santé sont les secteurs les moins affectés par la COVID-19. Si les emplois dans ces secteurs ont connu une évolution en dents de scie sur la période considérée, les pertes d'emplois ont diminué progressivement dans le secteur de l'éducation.

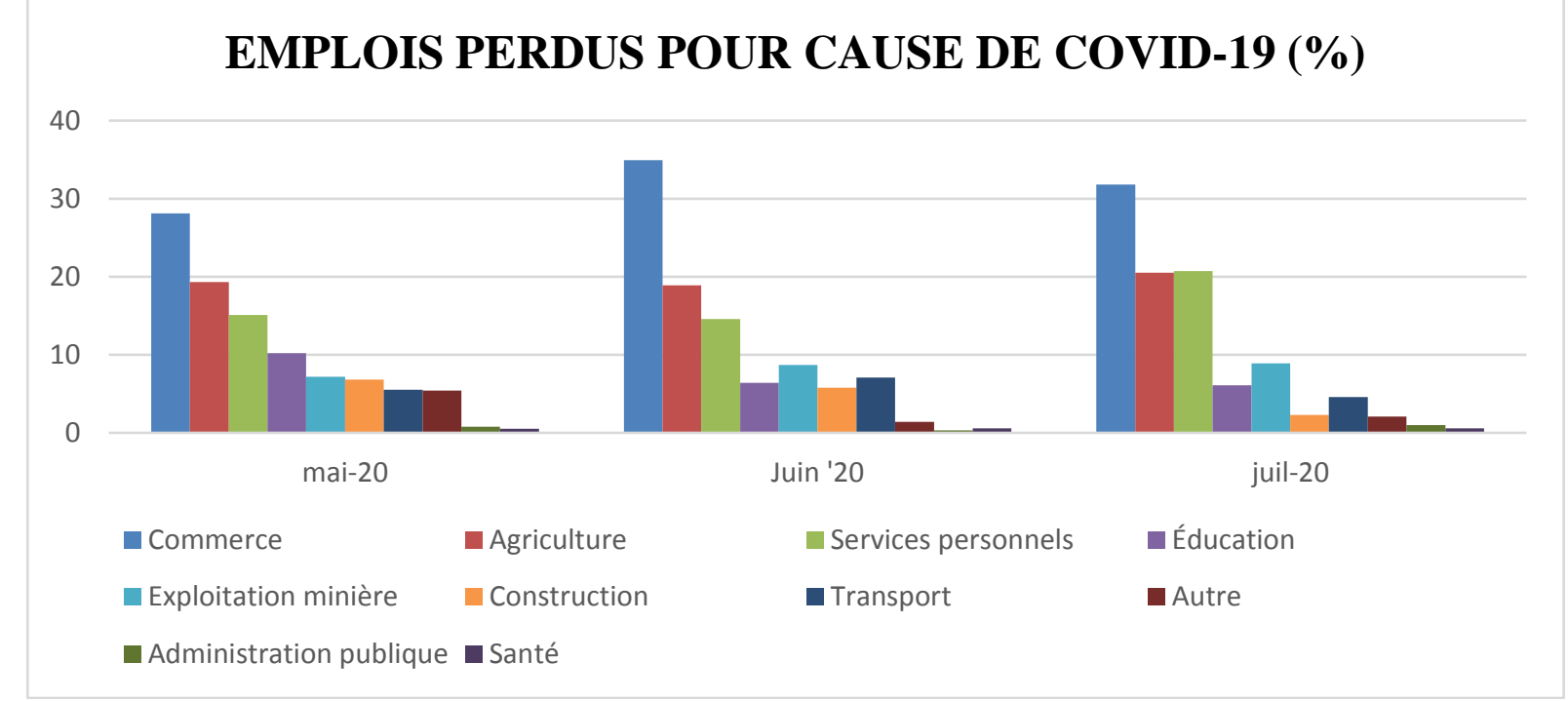

Source : calculs des auteurs sur la base des données HFS (2021)

\section{Graphique $\mathbf{n}^{\circ} 3$ : Evolution des emplois entre mai et juillet 2020}

\subsection{Accès aux services et soins de santé}

Le système sanitaire à la période pré-COVID était déjà vulnérable. Le Mali faisait partie déjà des cinq pays où la charge de morbidité et de mortalité est plus élevée (ONU-Mali, 2020). En effet, les dépenses publiques affectées à la santé représentaient seulement 5,4\% du budget, une proportion largement en dessous des engagements d'Abuja de porter la part des dépenses de santé dans le budget à $15 \%$. Cela amène les ménages à supporter une part importante des dépenses directes en soins de santé. Au même moment on note une répartition géographique inégale du personnel de santé à travers le pays et compte entre 2010 et 20184 infirmières, 1,4 médecin et 1 lit d'hôpital pour 10000 personnes contrairement aux normes recommandées par l'OMS (PNUD, 2020). Cette situation a fait que la pandémie a beaucoup pesé sur les résultats sanitaires et le système de santé malien par ses effets indirects, en imposant des demandes supplémentaires à un système de santé déjà fragile. Bien que la COVID-19 ait infecté moins de $1 \%$ de la population, avec un taux d'infection plus élevé observé dans les zones urbaines, par rapport aux zones rurales, les besoins en soins de santé non liés à la COVID ont augmenté de manière significative entre mai et octobre 2020 (HFS, 2021). Près de $80 \%$ des ménages ont déclaré avoir besoin de services de soins de santé en octobre 2020, contre seulement un tiers en mai 2020. Ces besoins en soins de santé ont quelque peu diminué en début de 2021 mais sont restés élevés, car plus de la moitié exprimait encore des besoins non satisfaits en soins de santé. Les cas de paludisme, de polio et 
d'autres maladies graves sont restés élevés et ont augmenté en 2020 et 2021 comparativement à 2019 (Cluster santé, 2021).

Si la pandémie en elle-même n'a pas significativement affecté l'accès aux services généraux de santé, certains ménages ont néanmoins déclaré un accès limité aux services de santé. Ces contraintes ont été observées particulièrement en mai et juin 2020. Elles étaient d'ordre financières et touchaient particulièrement les ménages pauvres et ceux dirigés par une femme. Selon l'analyse et la cartographie de la vulnérabilité mobile (mVAM) du Programme Alimentaire Mondial, entre $30 \%$ et $40 \%$ des ménages ont déclaré avoir des difficultés à accéder aux services de soins de santé depuis le début de la pandémie jusqu'en octobre 2020. Par ailleurs des perturbations dans les services de santé suite à la COVID-19 et à l'insécurité ont été déclarées par le Cluster Santé (ONU-Mali, 2020).

\subsection{Offre d'éducation et performance du système scolaire}

L'accès à l'éducation a connu une amélioration au cours de la décennie précédente suite aux efforts déployés par les autorités politiques (PNUD 2020). En 2018, près de la moitié des jeunes âgés entre 15 et 24 ans étaient analphabètes avec une proportion assez élevée chez les filles comparativement aux garçons (WDI, 2020). Environ $41 \%$ des filles en âge de fréquenter l'école primaire n'étaient pas inscrites dans une école primaire en 2018, contre 37,9\% des garçons. La pauvreté, le travail des enfants, les mariages précoces, l'accès limité aux écoles et les problèmes de sécurité sont quelquesunes des raisons qui expliquent les taux élevés d'abandon scolaire et de non-scolarisation au Mali (WDI, 2020). Cette situation éducative déjà fragile a été exacerbée par la fermeture des établissements scolaires en raison de la pandémie de la COVID-19. La mise en œuvre des mesures de riposte contre la COVID-19, a conduit à la fermeture des établissements scolaires de mars en septembre par les autorités gouvernementales, affectant ainsi, près de 3,8 millions d'enfants déjà en cours de scolarisation (UNICEF, 2020). Au plan alimentaire, la fermeture des établissements scolaires a affecté près de 750000 enfants des zones les plus pauvres qui ne bénéficiaient plus des programmes d'alimentation scolaire (ONU-Mali, 2020). En outre, les enquêtes de hautes fréquences de la Banque mondiale ont révélé plus de $64 \%$ déclarant que leurs enfants n'ont poursuivi aucune activité éducative. Ce taux a été de $67 \%$ pour les enfants des ménages vivant dans le milieu rural et $66,7 \%$ pour les enfants appartenant aux ménages pauvres. En revanche $28 \%$ des personnes touchées par l'enquête étaient engagées dans les activités d'apprentissage, $12,7 \%$ étudiaient en suivant des cours à domicile avec un membre du ménage, $28,2 \%$ travaillaient sur des exercices à travers des cours à domicile avec l'assistance d'un enseignant et $9,9 \%$ travaillaient sur des exercices fournis par des enseignants. Pour contrer les effets néfastes de COVID-19 sur le système éducatif, le gouvernement avait trouvé l'alternative d'offrir aux élèves et étudiants l'enseignement à travers les cours à la radio et la télé diffusé sur les chaines nationales et quelques chaines privées. Cependant, des inégalités géographiques dans l'apprentissage à distance par ces moyens numériques, notamment le visionnage d'un enseignement préenregistré en ligne, les applications mobiles, les programmes éducatifs à la télévision, la réalisation d'exercices en ligne donnés par les enseignants, la réalisation d'autres exercices en ligne, le visionnage de leçons à la télévision et la réception de leçons à la radio, ont été observés chez les enfants de Bamako et d'autres zones urbaines. Les cours à domicile étaient également plus répandus dans les zones urbaines par rapport aux zones rurales. Par ailleurs, il convient de rappeler que l'éducation avant la fermeture des écoles était affectée par les grèves des enseignants, responsables de la fermeture des écoles publiques avant la COVID-19 qui avait déjà touché plus de 2 millions d'élèves pendant plusieurs mois (UNICEF, 2020). De même que l'insécurité dans les zones 
de conflit qui a forcé la fermeture de plus de 1200 écoles au nord et au centre du pays (Nations Unies - Mali 2020), avec environ 403000 enfants affectés (1 344 écoles) tout au long de 2020 (UNICEF, 2020).

\subsection{Revenu, pauvreté et inégalités}

Selon le rapport (BAD, 2015), le revenu national par habitant au Mali avait enregistré une augmentation entre 2011 et 2015 pour atteindre 47,2\% avec une baisse consécutive du niveau de pauvreté qui s'était stabilisé autour de $42,7 \%$ en 2019 suite à la performance du secteur agricole (BAD, 2015). En revanche la répartition des revenus et de la pauvreté est restée inégale au sein du pays avec des taux plus élevés observés dans les communautés rurales du sud du pays. Le coefficient de Gini du Mali, de 33 entre 2010 et 2019, souligne le degré modéré d'inégalité des revenus dans le pays, par rapport à d'autres pays d'Afrique. Cette situation risque d'être inversée à cause des baisses significatives des sources de revenus qui ont été enregistrée pour la plupart des ménages.

Tant pour le milieu urbain que rural, chacun des milieux a connu une réduction pour les revenus non agricoles. Environ, $70 \%$ des ménages de Bamako et $67 \%$ des ménages des autres zones urbaines ont signalé une réduction des revenus liés aux activités non agricoles suite aux restrictions imposées lors de l'apparition de la COVID-19. Ainsi, 56\% des ménages ruraux ont constaté une réduction de leur revenu. Ces pertes de revenus sont en grande partie dues à la réduction de l'activité économique et de la demande de biens et service, imputable aux restrictions pour stopper la propagation de la pandémie. L'allègement des mesures de restriction a permis à de nombreux ménages d'améliorer le niveau de leur revenu. Les effets de la pandémie observés sur le revenu des ménages ruraux, qui tirent l'essentiel de l'agriculture résulte de la baisse de la productivité agricole. Selon les études de Koné et al (2020), l'accès limité aux intrants agricoles tels que les engrais par les producteurs maliens notamment ceux du coton et des céréales ont eu des effets négatifs sur les rendements agricoles. En outre, l'augmentation des prix des intrants a affectés la productivité des ménages, de même la réduction de l'offre de la main d'œuvre liée à la COVID-19 (Université de Wageningen et Institut royal des tropiques, 2020).

Les transferts des migrant est d'une importance capitale dans le cadre du financement du déficit du cycle de vie des individus. En effet, le financement du déficit de cycle de vie en 2019 était de 4057 milliards de francs CFA pour la tranche d'âge 0-29 ans et de 87 milliards les 71 ans et plus (ONDD, 2020). Cependant le ralentissement économique mondial a eu des implications majeures sur la réception des envois de fonds internationaux, qui constituent une importante source de revenus pour le Mali, en particulier pour les ménages pauvres et ruraux.

Une grande partie des envois de fonds internationaux sont destinés aux ménages des zones rurales et aux ménages pauvres, comme l'ont noté Chauvet et al. (2015). Tout choc sur cette source peut avoir des répercussions sur les capacités des ménages à faire face aux besoins qui se présentent en eux.

Il ressort de l'études des Nations Unies sur les effets socio-économiques de la COVID-19 au Mali, que $58,4 \%$ des ménages ruraux ont senti une baisse des envois de fonds internationaux. Cette proportion était de 49,9\% dans les ménages urbains. Il convient de noter que l'ampleur des réductions s'est quelque peu atténuée vers la fin de l'année 2020. La plupart des familles ont attribués la réduction des envois de fonds aux pertes d'emplois liés à la COVID-19. 


\subsection{Situation de l'emploi et moyens de subsistance}

Suite à la COVID-19, une grande partie des emplois est restée vulnérable, suite aux perturbations qu'elle a engendrées sur le marché du travail. Cependant, le taux de participation au marché du travail pour les personnes âgées de 15 ans et plus qui était d'environ $71 \%$ en 2019 n'a pas connue un véritable changement. Toutes fois, une différence prononcée subsiste entre les sexes avec $80 \%$ des hommes et $61 \%$ des femmes participant au marché du travail malien (OIT, 2020). Le secteur agricole est le plus grand employeur avec un taux de $62,4 \%$, suivi des services $30 \%$ et de l'industrie $7,6 \%$. Il existe également de grandes différences géographiques, les travailleurs urbains étant plus nombreux dans le secteur des services $71 \%$ contre $15,9 \%$ dans les zones rurales, et la plupart représentent les femmes $80 \%$. Le secteur informel semble employer une grande partie des travailleurs non agricoles, soit $90 \%$, et majoritairement dominé par les femmes 95\% (OIT, 2020). Les emplois vulnérables globalement comprennent des personnes qui travaillent pour leur propre compte et des travailleurs familiaux qui contribuent à l'emploi (OIT, 2018). En 2019, 81\% de ces emplois étaient classé vulnérables.

L'application des mesures barrières pour stopper la propagation de la COVID-19 ont eu un effet significatif sur les activités économiques et l'emploi. Les travailleurs urbains, les travailleurs les plus pauvres, les travailleurs des ménages dirigés par des femmes et le secteur des services sont les plus touchés. En effet, entre mai et juin 2020, environs 16,1\% des personnes qui travaillent à Bamako qui travaillaient avant l'apparition de la pandémie ont perdu leur emploi. Cette perte d'emploi est de $15,8 \%$ dans les autres régions urbaines et de $11,4 \%$ dans les zones rurales. Certaines études notamment celle de Weber et al. (2020), avaient déjà conclu que les effets des catastrophes sont plus importants dans les zones urbaines vue que les villes dépendent du secteur des services et des emplois du secteur informel des services comme le petit commerce, les magasins de détail, et autres qui subissent durement les effets des mesures de restriction liées à la pandémie et par la réduction de la demande (Weber et al. 2020).

En effet, la plupart des emplois perdus au cours de la période COVID relèvent du secteur des services pour environ 66\% (OIT, 2021). Elle a plus touché les activités commerciales. Par ailleurs quelques perturbations ont été observées dans le secteur agricole pour environ $20 \%$. Les familles qui ont été les plus affectées sont celle dirigées par les femmes et celles qui étaient déjà dans la tranche des pauvres. Ainsi les enquêtes au niveau national ont révélé que 43,6\% des ménages ont mentionné au moins une perte d'emploi dont les motifs reposaient sur la COVID-19 en mai 2020.

Depuis mai 2020, la proportion des personnes sans emploi a diminué progressivement en avec l'assouplissement des mesures, la proportion des personnes sans emploi suit une réduction de son taux. Il est passé de 43,6\% au moment de l'application des restrictions, à environ 1,2\% en janvier 2021 avec l'allègement des mesures restrictives. Néanmoins les villes comme Bamako restent encore touchées par les effets de la COVID-19. Le manque de client est l'une des raisons principales auxquelles peuvent être ajoutée, les mauvaises ventes, la difficulté à acquérir des intrants/matières premières, la réduction de l'offre de travail et la réduction des heures de travail. En ce qui concerne les exploitants des entreprises familiales plus de deux tiers d'entre elles annoncent avoir des revenus inférieurs à la celle de période pré-COVID-19. Par ailleurs les causes principalement évoquées des pertes d'emplois reviennent à la COVID-19 à travers l'application des mesures barrières. Les maladies et les manques d'activités sont moins évoqués comme motifs de pertes d'emploi. 


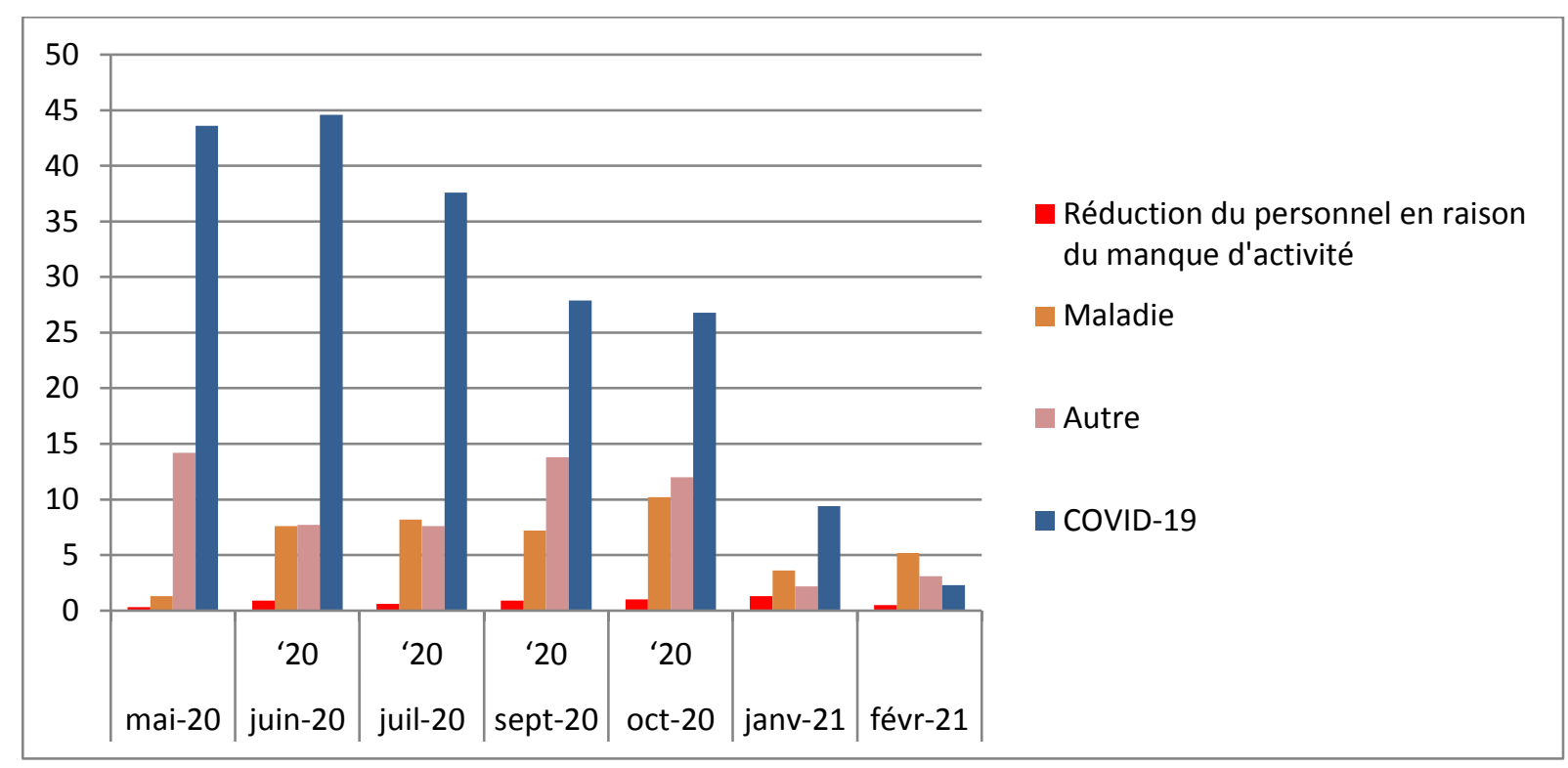

Source : calculs des auteurs sur la base des données HFS (2021)

\section{Graphique ${ }^{\circ} 4$ : Causes des pertes d’emplois des personnes en emploi avant la COVID-19}

\subsection{Sécurité alimentaire et nutrition}

Selon le rapport du programme alimentaire mondial, l'insécurité alimentaire et la malnutrition sont d'une forte prévalence au Mali, particulièrement les ménages affectés par la pauvreté et ceux vivant dans le milieu rural. Environ un enfant de moins de cinq ans sur quatre souffrait de dénutrition chronique. Les effets cumulés des catastrophes naturelles fréquentes (sécheresse, inondations), de la violence armée et des conflits, des déplacements, de la pauvreté persistante, du changement climatique et des défaillances du marché lié à l'économie agricole, figurent au rang des facteurs qui limitent les progrès en matière de sécurité alimentaire dans le pays au fil du temps. L'intensification des conflits et des tensions communautaires et les déplacements de populations qui en découlent sont restés un facteur majeur d'insécurité alimentaire et nutritionnelle dans les régions du nord et du centre du Mali (Cluster sécurité alimentaire 2021).

La COVID-19 a aggravé l'insécurité alimentaire au Mali par la baisse des revenus des ménages suite à la réduction des activités économiques et à la diminution des envois de fonds. En outre, le rapport Fews Net (2021) indique l'accès limité aux marchés, les perturbations des chaînes d'approvisionnement alimentaire nationales et régionales, l'augmentation des coûts et la baisse de la disponibilité des intrants agricoles constituent aussi quelques facteurs exacerbant l'insécurité alimentaire (FEWS NET, 2021). Par ailleurs, il ressort du rapport (FMI, 2021) que les hausse des prix des denrées alimentaires ont été observé consécutifs au début de la pandémie, ce qui a constitué un choc de revenu pour $25 \%$ de la population (HFS, 2021). Environ, 47,8\% des ménages ont déclaré que la COVID-19 a eu un effet sur leur capacité à satisfaire leurs besoins alimentaires. Les premières données empiriques affirment que la pandémie a augmenté la probabilité d'être en situation d'insécurité alimentaire par rapport à la période pré-pandémique de 4 points de pourcentage.

Les travaux d'Adjognon et al. (2020) montrent que l'augmentation de la probabilité d'insécurité alimentaire pour les ménages urbains est environ deux fois plus élevée que pour les ménages des zones rurales (FEW NET, 2021). De même que pour le rapport de l'enquête à haute fréquence, il ressort que les ménages dans le milieu urbain attribuent à la COVID-19 leur situation d'insécurité alimentaire 
comparativement aux ménages ruraux. Cet effet ressenti par les ménages urbains est lié à l'augmentation du prix des principaux aliments consommés en milieu urbain. Cependant, il convient de noter que le niveau de sécurité alimentaire s'est amélioré vers la fin de 2020. Cela revient du témoignage de plus de $95 \%$ des ménages qui ont déclaré pouvoir accéder à la plupart des produits de base. Cette amélioration peut être attribuée au-delà de la bonne saison agricole à l'assouplissement des restrictions de confinement liées à la pandémie après la première vague de la COVID-19. En revanche, la situation de la sécurité alimentaire s'est toutefois dégradée au début de l'année 2021 suite aux facteurs combinés liées aux effets combinés du conflit, de l'insécurité, de l'augmentation des prix de certains aliments de base, de la réduction de l'offre de travail, des revenus et des revenus (SAP, 2021). Cette situation révèle que la pandémie a aggravé les vulnérabilités socio-économiques existantes à travers les chocs qu'ont subi les ménages, entre autres l'augmentation du prix des principaux aliments consommés et celui des intrants agricoles, la perte des emplois et les faillites des entreprises familiales. Globalement, les ménages ayant subi un ou plusieurs chocs engendrés par la COVID-19 est estimé à $62 \%$ entre mars et juin 2020. La hausse des prix des denrées alimentaires et le manque d'intrants agricoles constituent les chocs les plus répandus parmi les ménages pauvres et ruraux, tandis que les pertes d'emploi et les faillites d'entreprises familiales étaient plus fréquentes dans les zones urbaines.

\section{Mécanisme de résilience :}

Les principales stratégies d'adaptation utilisées par les ménages pour faire face aux chocs comprenaient la vente d'actifs, l'engagement dans des activités génératrices de revenus supplémentaires, le recours à l'épargne, l'aide des amis et de la famille, la réduction de la consommation alimentaire et l'emprunt auprès des amis et de la famille. Par rapport à ces stratégies d'adaptation, les ménages ruraux étaient plus susceptibles de recourir à la vente d'actifs, tandis que les ménages urbains en dehors de Bamako s'engageaient dans des activités génératrices de revenus supplémentaires. En outre, les ménages de Bamako étaient plus susceptibles de recevoir de l'aide de leurs amis et de leur famille ou de réduire leur consommation alimentaire. Alors que les ménages pauvres ont eu recours à la vente de leurs actifs, les ménages non pauvres se sont appuyés de manière significative sur leur épargne et l'aide de leurs amis et de leur famille. Cependant environ, $35 \%$ des ménages ayant subi plusieurs chocs depuis le début de la pandémie n'ont eu recours à aucune stratégie par manque de ressources. Le recours à ces différentes stratégies peut avoir des conséquences négatives en termes de transitions de la pauvreté vue l'incapacité de ces ménages à faire face aux chocs sur le revenu et l'épargne.

Dans le cadre de la protection sociale, les filets de sécurité sociale ont joué un rôle important dans l'atténuation des effets des chocs sur les différents ménages. Cependant, moins de $20 \%$ de la population était couverte par les politiques de protection sociale avant le début de la pandémie (Nations Unies Mali, 2020). Ce faible niveau de la couverture sociale peut être imputable à la faible capacité des ressources publiques pour étendre la protection à une couche large de la population. Cette insuffisance révélée dans les stratégies gouvernementales confère le pouvoir à certaines ONG internationales, agences des Nations Unies et acteurs nationaux de s'engager dans l'assistance sociale (O'Brien et al. 2018). Selon les résultats des enquêtes HFS, seulement $4 \%$ des ménages ont reçu une forme d'aide sociale en juin 2020, dont la majorité était une aide alimentaire en nature (77\%) (Gentilini et al. (2020). D'autres réponses sociales ont inclus les transferts monétaires d'urgence COVID-19 en faveur de 75000 ménages repartis entre Bamako et les autres régions, l'exonération des services publics pour les mois d'avril et mai 2020 pour les ménages vulnérables, certaines exonérations fiscales temporaires d'avril à juin 2020 sur les importations de riz et de lait, et la TVA sur les factures d'électricité et d'eau, la 
suspension temporaire de l'impôt sur le revenu simplifié et des taxes foncières jusqu'en décembre 2020 (FMI, 2020). Le volet fiscal consistait à alléger les charges fiscales de près de $0,6 \%$ du PIB. Le volet santé portait sur un plan d'urgence et l'achat des de fournitures pour un montant d'environ $0,5 \% \mathrm{du}$ PIB, un soutien aux ménages vulnérables pour $1,2 \%$ du PIB et un soutien aux entreprises pour $0,5 \% \mathrm{du}$ PIB (FMI, 2021). D'autres mesures, telles que les exonérations de taxes et de droits d'importation, ne profitent pas à de larges segments de la population, en particulier ceux de l'économie informelle.

\section{Conclusion}

Cette étude sur les effets de la COVID-19 et les mécanismes de résiliences des ménages avait pour objectif de faire une évaluation des effets de la pandémie sur le revenu, la consommation des individus ainsi que sur l'accès aux services sociaux de base comme l'éducation, la santé et la résilience des ménages. Elle a permis de montrer que la COVID-19 a eu une forte influence négative du tissu socioéconomique qui était déjà fragilisé par une instabilité politique et sécuritaire. Globalement, elle a affecté l'économie malienne à travers la réduction de la demande sur le marché, la volatilité des prix des produits de base et des matières premières et la perturbation de la chaîne d'approvisionnement. Il ressort des analyses que les effets négatifs de la COVID-19 ont été substantiellement ressenti au niveau des secteurs du commerce, de l'agriculture, le commerce, le tourisme et le transport à travers les pertes d'emplois et la réduction des revenus. Ces effets de la pandémie observés sur les ménages et leurs moyens d'existences, pourraient compromettre les progrès déjà réalisés dans l'atteinte des Objectifs du Développement Durable, particulièrement dans le domaine de la réduction de la pauvreté, de la sécurité alimentaire, de l'emploi, de l'éducation et de la santé. Ces résultats auxquels nous sommes parvenus confirment certains travaux antérieurs notamment celui de la Banque mondiale réalisée par Kakpo et al (2021) selon lequel, la pandémie a entrainé une réduction de la croissance qui est passé d'une croissance moyenne de 5,1\% entre 2013 et 2019 à 2\% en 2020. La même étude avait conclu une réduction de la consommation privée suite à la baisse des envois de fonds et les mesures de confinement. Ils confirment aussi les résultats de YAN (2021) selon lesquels, la crise sanitaire a provoqué une augmentation du nombre des pauvres de près de 900000 personnes en donnant un coup de frein aux progrès réalisés au cours de la dernière décennie dans les efforts de la réduction de la pauvreté. Les implications en termes de politique consisteront à étendre les mesures gouvernementales dans le domaine de la protection sociale aux couches les plus vulnérables suivant un critère de ciblage et une coordination entre les acteurs au développement; à renforcer les politiques en matière d'éducation et de soutien au secteur privé et à assurer un meilleur équilibre entre les dépenses sociales et les besoins d'investissements.

\section{BIBLIOGRAPHIE}

[1] Adjognon, G. S., Bloem, J. R., \& Sanoh, A, "La pandémie de coronavirus et la sécurité alimentaire : Evidence from West Africa", Document de travail de recherche sur les politiques de la Banque mondiale, 2020

[2] BAD "Perspectives économiques en Afrique ", Banque Africaine de Développement, 2015

[3] Banque mondiale "Global Economic Prospect", Groupe de la Banque mondiale, janvier 2021. Disponible sur : https://www.worldbank.org/en/publication/global-economic-prospects

[4] Chauvet, L., Gubert, F., Mercier, M., \& Mesplé-Somps, S. "Les associations de quartier d'origine des migrants et le développement local au Mali".,The Scandinavian Journal of Economics, 117(2), 2015, pp. 686-722,.

[5] Cluster Santé Mali. "Maladie à déclaration Obligatoire (MADO)". Bulletin cluster santé, Janvier - Mars 2021.

[6] Cluster sécurité alimentaire du Mali, "Note de plaidoyer en faveur d'une réponse coordonnée et mise à l'échelle de sécurité alimentaire et nutritionnelle dans le cadre du covid-19 au mali", Cluster Sécurité Alimentaire du Mali, 2020. 
[7] FEWS NET, "Accès limité à la nourriture pour les ménages dans certaines localités en raison des conflits et du COVID-19", Perspectives de la sécurité alimentaire, Février 2021, https://fews.net/west-africa/mali/food-securityoutlook/february-2021

[8] FMI, "Mali requests for disbursement under the rapid credit facility and rephasing of access under the extended credit facility arrangement- press release ; staff report ; and statement by the executive director for Mali". FMI. Country Report No. 20/153 ; 2020, p. 44.

[9] FMI, "Mali deuxième et troisième révisions au titre de l'arrangement de facilité de crédit étendu", Rapport pays du FMI $n^{\circ} 21 / 67,2021$.

[10] FMI, "Reviews of the extended credit facility agreement", IMF report no 21/67, mars 2021. Disponible sur : https://www.imf.org/fr/Countries/ResRep/MLI\#a2

[11] Gentilini, U., Almenfi, M., \& Dale, P., "Réponses de la protection sociale et de l'emploi au COVID-19 : Un examen en temps réel des mesures prises par les pays", Banque mondiale, 2020, https://www.imf.org/en/Topics/imf-andcovid19/Policy-Responses-to-COVID-19\#M , (Consulté le 19 juin 2021)

[12] Michael, W. Amparo, P. L, Ivette, Maria, C. G. "Implications du COVID-19 sur le marché du travail dans quatre pays africains", Blogs de la Banque mondiale. Novembre, 2020, https://blogs.worldbank.org/opendata/labor-marketimpacts-covid-19-four-african-countries (consulté le 30 avril 2020)

[13] Nations Unies, "Analyse rapide des impacts socio-économiques du COVID-19 au Mali", UN Mali, mai 2020.

[14] O'Brien, C., Congrave, J., \& Barca, V. "Protection sociale et réponses humanitaires à l'insécurité alimentaire et à la pauvreté au Mali", Oxford Policy Management Policy Brief, 8 ; 2018.

[15] OIT "Emploi rémunéré vs emploi vulnérable", ILOSTAT, Pleins feux sur les statistiques du travail, n 3, 2018.

[16] OIT "COVID-19 et le monde du travail", Septième édition, Organisation internationale du travail, 2021

[17] ONDD-Mali "Rapport de synthèse de suivi du dividende démographique", ONDD-Mali, 2020.

[18] ONU Mali. "Analyse rapide des impacts socio-économiques du COVID-19 au Mali"; Bulletin du cluster santé, $n^{\circ} 1$ 2021 Janvier-Mars 2021, Cluster Santé Mali.

[19] PNUD, "Préparation et vulnérabilité aux tableaux de bord COVID-19 COVID-19 et développement humain", 2020, https://www1.undp.org/content/undp/fr/home/news-

centre/news/2020/COVID19_UNDP_data_dashboards_reveal_disparities_among_countries_to_cope_and_recover.ht $\mathrm{ml}$, (consulté le 16 juin 2021)

[20] PNUD, "Rapports sur le développement humain", 2020, Consulté le 22 mars 2021, à l'adresse http://hdr.undp.org/en/countries/profiles/MLI.

[21] SAP, "Résultats de l'analyse des résultats mars 2021 mali", 2021 https://fscluster.org/sites/default/files/documents/presentation_resultats_oa_mars_2021.pdf

[22] UNICEF, "Rapport sur la situation humanitaire au Mali", UNICEF, No12, 2020

[23] World Bank, "Mali note sur la situation économique : Protéger les personnes vulnérables le temps de la reprise", World Bank, Washington, DC, 2021

[24] Y., Xun, "Comprendre l'impact de la COVID-19 pour mieux agir», World Bank, Washington, DC, 2021 